\title{
Lückenfinder statt Lückenbüßer
}

\author{
Unternehmerinnen oder: \\ das tägliche Geschäft in der Sozialen Arbeit
}

Anne Klüser

Social work can be organised in an ideal way in the form of self-employment - a form that goes hand in hand with the identity of the profession. This form matches the perception and action perspective of social workers and social education workers in an ideal way.

Le statut d'indépendant s'adapte idéalement au travail social - un statut qui va de pair avec l'essence même de la profession. Elle correspond parfaitement à la perception et à la perspective d'action des et assistants sociaux socio-éducatives.

Dr. Anne Klüser ist DiplomSozialarbeiterin und Diplom-Pädagogin. Sie arbeitet als freiberufliche gesetzliche Betreuerin. An der Katholischen Fachhochschule Nordrhein-Westfalen, Abteilung Köln, ist sie zudem als Lehrbeauftragte und als Referentin für die Fort- und Weiterbildungsabteilung tätig. E-Mail anne.klueser@ngi.de
Soziale Arbeit lässt sich in Form unternehmerischer Selbständigkeit ideal und identitätskonform organisieren und entspricht der Auffassungs- und Tätigkeitsweise von Sozialarbeiterinnen und Sozialpädagoginnen* besonders gut.

Von unternehmerischer Sozialer Arbeit kann man schon immer sprechen. Nur hätte der Begriff früher Probleme aufgeworfen; vornehmlich wahrscheinlich ideologische, indem man Unternehmen mit der Privatwirtschaft und mit Geldverdienen in Verbindung brachte, die Soziale Arbeit aber keinesfalls. Seit »Marktelemente " in die Sozialbürokratie und die Organisationen der freien Wohlfahrtspflege Einzug gehalten haben, findet man selbst bei kirchlichen Trägern eine »Unternehmensphilosophie " oder eine Aufteilung in »Unternehmensbereiche«.

\section{Zum Begriff des Unternehmers}

In einem Beitrag zur Frage, ob und wie eine Ausbildung zum Unternehmer möglich ist, beschreibt Baecker (1999) Unternehmer als »Lückenfinder «. "Lücken « sind Möglichkeiten. Die Lücken selbst kann man dem künftigen Unternehmer nicht beibringen, sondern nur Fragen, die zu Lücken führen. Unternehmer sind darüber hinaus »Leser unseres Lebensstils «. Sie operieren als Beobachter zweiter Ordnung, die nicht nur sich selbst und ihre Umwelt beobachten, sondern auch, wie diese Umwelt beobachtet.

Erfolgreiche Unternehmer weisen ein besonderes Kommunikationsgeschick auf, das nicht vollständig lehrbar ist. Und sie haben, wenn sie intervenieren wollen, nicht nur mit dem Unternehmen, sondern

\footnotetext{
* Zur besseren Lesbarkeit wurde in Überschrift und Text meistens auf die Ergänzung der männlichen Form verzichtet. In der Sozialen Arbeit sind ja ganz überwiegend Frauen beschäftigt.
}

immer auch mit sich im Unternehmen zu tun. In letzter Zeit, da ob gestiegener Unsicherheit und Komplexität herkömmliche Verfahren der Kontrolle und Erfassung versagen, wird offensichtlicher, dass der Versuch, Unternehmertum mit Hilfe der Betriebswirtschaft rational zu lehren, gescheitert ist, ja die Betriebswirtschaftslehre das Unternehmertum eher "gezähmt « hat.

Baecker beschränkt sein Unternehmerverständnis weder auf Selbstständige im eigentlichen Sinne noch auf die Privatwirtschaft mit primär gewinnorientierter Ausrichtung. Vielmehr betrifft es auch den gesamten Non-Profit-Bereich und Angestellte, von denen unternehmerisches Handeln im genannten Sinne erwartet wird (»Intrapreneurship «).

Unter dem Begriff »Arbeitskraftunternehmer « problematisierten Pongratz/Voß (1998) bereits vor zehn Jahren einen neuen Typus des Arbeitnehmers. Dieser ist im Vergleich zum traditionellen, eher reaktiv agierenden Beschäftigten aktiver Unternehmer seiner selbst. Seine wesentlichen Merkmale sind "Selbst-Kontrolle (verstärkte selbstständige Planung, Steuerung und Überwachung der eigenen Tätigkeit), Selbst-Ökonomisierung (zunehmende aktiv zweckgerichtete >Produktion< und >Vermarktung ‘ der eigenen Fähigkeiten und Leistungen - auf dem Arbeitsmarkt wie innerhalb von Betrieben), Selbst-Rationalisierung (wachsende bewusste Durchorganisation von Alltag und Lebensverlauf und Tendenz zur Verbetrieblichung von Lebensführung) « (Prongratz/Voß 2003). Sie sehen eine starke Annäherung des »Unternehmers der eigenen Arbeitskraft «, der formal angestellt ist, zum selbstständigen Unternehmer.

Während hier der Fokus auf verschiedene kritische Aspekte der »neuen Stufe der marktbasierten Ökonomisierung von Arbeitskraft « gelegt wird, betont Pankoke die Potenziale und Chancen unternehmerischen Denkens und Handelns als 
Intrapreneurship einerseits, aber insbesondere auch im Hinblick auf die radikale Entscheidung zur Gründung eines eigenen Unternehmens als Lebensperspektive andererseits (Pankoke/te Neues 2000): »Wohl aus keiner Position in der Gesellschaft ergeben sich solche Chancen freier Entscheidung. " Unternehmertum heißt Risikogestaltung in komplexen Feldern, wo Handeln durch bürokratische Routinen und einfache Entscheidung an seine Grenzen stößt. Unternehmer-Dasein beschränkte sich nie auf bloße Profitmaximierung, sondern wollte immer auch mit »Unternehmergeist « etwas bewegen, bezog sich also insofern auch auf gesellschaftliche Entwicklungspotenziale (vgl. ebd.).

\section{Unternehmerische Soziale Arbeit}

Im oben verstandenen Sinne sind Sozialarbeiterinnen und Sozialpädagoginnen im Kern Unternehmerinnen und Unternehmer.

- Die in Ratgebern zur Existenzgründung in der Sozialen Arbeit oft anhand von Eigenschaftsauflistungen konstatierten Gründungsvorteile von Sozialarbeiterinnen und Sozialpädagoginnen finden ihr Pendant in Studienordnungen (neben Fachkompetenz insbesondere sogenannte "Schlüsselqualifikationen « und »soft skills «).

- Sozialarbeiterinnen und Sozialpädagoginnen sind »Grenzgänger « (Pankoke). Vermitteln unter komplexen Bedingungen auf und zwischen verschiedenen Ebenen ist eine ihrer wesentlichen Aufgaben. Auf der Ebene der Arbeitsorganisation tragen dem selbst Behörden Rechnung. Sozialarbeiterinnen und Sozialpädagoginnen haben und benötigen sehr viel mehr Freiheit als Angestellte oder Beamte anderer Bereiche, beispielsweise hinsichtlich der Dienstzeiten, Anwesenheitszeiten, Gleitzeiten etc., da ihre Arbeit flexiblen Einsatz, teils Überstunden im Akutfall, erfordert. Ein nicht unerheblicher Teil der Tätigkeit wird im Rahmen von Außendienst (Hausbesuche etc.) erbracht.

- Soziale Arbeit ist visionsfrei nicht denkbar und unpersönlich und unter Abschaltung von Leidenschaft und Enthusiasmus nicht möglich. Sozialarbeiterinnen und Sozialpädagoginnen sind qua ihrer Aufgabe immer Querdenkerinnen. Eine ihrer Aufgaben ist es, Möglichkeiten zu schaffen, also »Lücken « zu finden für Menschen, denen es an eigenen Ressourcen fehlt und bei denen allgemein übliche Mechanismen und Sicherungen versagen. Als professionelle »Lückenfinderinnen " sind sie per se Unternehmerinnen. Sofern sie diese Lücken im Rahmen institutioneller Arrangements finden, sind sie Intrapreneurinnen.

- Konstitutives Merkmal Sozialer Arbeit ist Bewegung. Dies betrifft die Ebene ihrer eigenen Existenz, die teils aus gesellschaftlichen Bewegungen entstanden ist und sich durch solche heute
Auftragsvergabe prinzipiell gleich zu behandeln, sofern sie die für alle gültigen Voraussetzungen erfüllen. Immer mehr Bereiche werden privatisiert, zuletzt die gesamte Bewährungshilfe in Baden-Württemberg. Berufliche Selbständigkeit ist in vielen Bereichen Sozialer Arbeit bereits möglich und in den meisten vorstellbar. Die Zahl der Selbstständigen steigt offensichtlich (wobei allerdings eine genaue Statistik fehlt). So wird beispielsweise die Berufsbetreuung ( $\mathbb{S} 1986 \mathrm{ff}$. BGB) fast vollständig in beruflicher Selbständigkeit erbracht. Auch in anderen Bereichen, beispielsweise der Jugendhilfe, haben sich Selbstständige dauerhaft etabliert. mindestens anreichert, sowie das Bewegen von Gesellschaft und Individuen durch ihr eigenes Wirken.

- Sozialarbeiterinnen und Sozialarbeiter, Sozialpädagoginnen und Sozialpädagogen sind seit jeher »Leser unseres Lebensstils «. In ihrem traditionellen Methodenspektrum sind Methoden der Beobachtung und insbesondere der Beobachtung zweiter Ordnung in Form von Praxisberatung, kollegialer Beratung, Intervision, Supervision und Evaluation installiert und institutionalisiert. Es ist die Aufgabe Sozialer Arbeit, den »gelesenen Lebensstil « zur Grundlage von Interventionen und zur Basis der Anregung von Prozessen zu machen. Zu diesem Zweck stehen ebenfalls Methoden sowie Techniken zur Verfügung.

\section{Unternehmerische Selbständigkeit}

Eine der für die Selbständigkeit in der Sozialen Arbeit grundlegenden Veränderungen ist der Fall des Vorrangs der freien Wohlfahrtspflege. Die Sozialverwaltungen haben Leistungserbringer bei der
Es stellt sich die Frage, warum Sozialarbeiterinnen und Sozialpädagoginnen berufliche Selbständigkeit wählen? Dass dies nur ein Weg ist, prekären und instabilen Verhältnissen bei traditionellen Trägern auszuweichen oder, wie vielfach vermutet wird, (drohender) Arbeitslosigkeit zu entgehen, ist unwahrscheinlich. Engel (2000) identifizierte zumindest eine Gruppe von Selbstständigen, die auf eine langjährige Tätigkeit in der freien Wohlfahrtspflege zurückblicken und mit wachsender Berufserfahrung Defizite wahrnehmen. Diese betreffen sowohl den eigenen Status und die Honorierung der eigenen Arbeit als auch inhaltliche und Entwicklungsfragen. Sie sehen sich durch die Institution eher eingeschränkt in ihrem Gestaltungspotenzial, leiden unter Arbeitsroutinen und erfahren, dass selbst initiierte Weiterbildung und Zusatzausbildung nicht nur nicht honoriert wird, sondern zusätzlich erworbene Kompetenzen sogar Probleme aufwerfen. Selbständigkeit ist für sie ein Versuch, Unzufriedenheit mit der bisherigen Arbeitssituation zu überwinden.

Trube (2000) verweist auf Widersprüche, wenn Soziale Arbeit entgegen ihrer Identität organisiert wird. Freie Wohl- 
fahrtspflege und Sozialbürokratie als Institutionen sehen sich mit dem Vorwurf des Korporatismus konfrontiert und stehen heute in vielerlei Hinsicht nicht mehr für Unternehmertum und Bewegung.

Eine Antwort auf die Frage nach der Attraktivität selbstständiger Sozialer Arbeit könnte daher sein: Soziale Arbeit lässt sich in Form unternehmerischer Selbständigkeit ideal und identitätskonform organisieren und entspricht der Auffassungs- und Tätigkeitsweise von Sozialarbeiterinnen und Sozialpädagoginnen besonders gut.

Unternehmerische Selbständigkeit stärkt insofern über Authentizität rekursiv die Identität Sozialer Arbeit. Erfolgreiche selbstständige Sozialarbeiterinnen und Sozialpädagoginnen zeichnen sich

\section{Literatur}

Baecker, D. 1999: Das Handwerk des Unternehmers, in: Baecker, D. 1999: Organisation als System, Frankfurt am Main, S. 330-377.

Engel, A. 2000: Selbstständige Soziale Arbeit, in: Blätter der Wohlfahrtspflege 7+8, S. 166-169.

Klüser, A. 2006: Zum Verhältnis von Sozialer Arbeit und Betreuung unter besonderer Berücksichtigung beruflicher Selbständigkeit. Verberuflichungs- und Professionalisierungsprozesse am Beispiel freiberuflicher Betreuungsführung in Köln, Duisburg.

Pankoke, E. 2000: Sozialethiken und Wohlfahrtskulturen. Grenzen und Schwellen wohlfahrtsstaatlicher Modernität, in: Prisching, M. (Hg.) 2000: Ethik des Sozialstaats, Wien, S. 13-36.

Pankoke, E./te Neues, E. 2000: Unternehmerisches Denken und Handeln als Lebensperspektive, Hagen.

Pongratz, H. J./Voß, G. G. 1998: Der Arbeitskraftunternehmer. Eine neue Grundform der Ware Arbeitskraft?, in: Kölner Zeitschrift für Soziologie und Sozialpsychologie 50, S. 131-158.

Pongratz, H. J./Voß, G. G. 2000: Arbeitskraftunternehmer. Erwerbsorientierungen in entgrenzten Arbeitsformen, Berlin.

Trube, A. 2000: Freiheit oder Sicherheit. Die neue Selbständigkeit und die neue Sozialarbeit, in: Sozial extra 3, S. 26-32. dadurch aus, dass bestimmte Fähigkeiten besonders ausgeprägt sind (vgl. Engel a.a.O.): Sie besitzen sehr gute Fach- und Organisationskenntnisse und viel Kreativität bei der Lösung von Problemen. Sie sind besonders vorausschauend und agieren eher, bevor sie zu Reaktionen in Bezug auf Existenz gefährdende Situationen gezwungen sind. Sie verfügen über eine realistische Selbsteinschätzung und eine enorm ausgeprägte Selbstreflexion. Auf dieser Basis arbeiten sie konsequent an eigenen Defiziten. Die Existenz wird darüber hinaus so angelegt, dass die Abhängigkeit von unbeeinflussbaren Rahmenbedingungen gering gehalten wird: Das Tätigkeitsspektrum wird eher breit gefächert, ohne dass das spezifische Profil abhanden kommt. Und es werden vermehrt Leistungen in das Tätigkeitsspektrum einbezogen, an denen die Privatwirtschaft Interesse zeigt und die nicht aus öffentlichen Mitteln bezahlt werden.

\section{Wie weiter?}

Natürlich ist noch einiges zu tun. Sozialarbeiterinnen und Sozialpädagoginnen müssen lernen, Geld zu verdienen und zu kalkulieren, ohne sich dem Profit zu ver-

\section{Beispiel Selbstständigkeit: Supervision und Beratung}

Seit zehn Jahren bin ich in Hamburg selbstständig. Als Sozialarbeiterin qualifizierte ich mich berufsbegleitend zur Familientherapeutin und anschließend zur systemischen Supervisorin.

Der Schwerpunkt als Honorarkraft in der sozialpädagogischen Familienhilfe (mit extrem niedrigem Stundensatz) erleichterte mir den Start. Die Aufträge für Supervision und Coaching wuchsen, zunächst im Bereich der Jugendhilfe, meiner Feldkompetenz, später in allen sozialen Bereichen und darüber hinaus kamen Aufträge beispielsweise für eine Fortbildung zu kundenorientiertem Arbeiten in behördennahen Einrichtungen.

Zunächst genügten mir ein Büro und ein Arbeitszimmer im eigenen Haus, besonders als die Zimmer der beiden Töchter frei wurden. Seit diesem Jahr verfüge ich über ein angemietetes Beratungszimmer in der Nähe der Wohnung.

Ich genieße die vielfältigen Aufträge in verschiedenen Arbeitsbereichen, die interessanten Menschen und das Abenteuer am Arbeitsplatz. Geärgert hatte mich alle Vierteljahr der Büroaufwand für die Umsatzsteuervorauszahlungen und die Diskussionen mit Behörden, die mir keine Umsatzsteuer zahlen.

Mit dem neuen Büro habe ich begonnen, verstärkt bei mir Supervisionen zu machen und nicht in den Einrichtungen. Das verbessert die Arbeitsatmosphäre und ist für mich entspannend.

Eine große Hilfe war immer die Intervisionsgruppe mit Kolleginnen und Kollegen sowie die ermutigende Einzelsupervision. Die Vernetzung in den Fachgruppen des Berufsverbandes für Soziale Arbeit gab mir Anregungen aus anderen Bundesländern. Ich arbeite sehr gern engagiert und bin froh, dass ich den Schritt in die Selbständigkeit gewagt habe. 